Pacific Journal of Mathematics

INTERTWINING OPERATORS BETWEEN 


\title{
INTERTWINING OPERATORS BETWEEN HOLOMORPHICALLY INDUCED MODULES
}

\author{
Brian D. Boe and David H. Collingwood
}

\begin{abstract}
Let $(G, K)$ be an irreducible hermitian symmetric pair of non-compact type. The authors study the spaces of covariant differential intertwining operators between the continuations of holomorphic discrete series for this pair. In particular, when $G=\mathrm{SU}(p, q), \operatorname{Sp}(n, \mathbf{R})$, or $\mathrm{SO}^{*}(2 n)$, an algorithm is provided to reduce the problem for regular integral infinitesimal character to that for semiregular representations. In case $G=\mathrm{SU}(p, q)$, this latter problem is solved using an equivalence of categories due to Enright-Shelton (providing a reduction to the regular case for a lower rank group).

In addition, an explicit description of the non-zero spaces of intertwining operators is given for the two exceptional cases, $E_{6}$ and $E_{7}$. The remaining case, $G=\mathrm{SO}(2, n)$, has been treated previously. All results are obtained in the (dual) setting of homomorphisms between generalized Verma modules.
\end{abstract}

1. Introduction. Let $(G, K)$ be an irreducible hermitian symmetric pair of non-compact type; that is, $G$ is a real Lie group and $K$ a maximal compact subgroup, such that $G / K$ is a hermitian symmetric space. Associated to this pair is a family of holomorphically induced representations, the continuation of holomorphic discrete series. This family has been investigated from many points of view, by numerous people. In the present note, we study the spaces of covariant differential intertwining operators between these representations. In particular, it is known from a previous work [3] that, in the integral case, these spaces are at most one-dimensional; thus, the question is reduced to one of existence. Our main result is an algorithm for reducing the problem for regular integral infinitesimal character to that for semiregular modules, when $G=$ $\mathrm{SU}(p, q), \mathrm{Sp}(n, \mathbf{R})$, or $\mathrm{SO}^{*}(2 n)$. In case $G=\mathrm{SU}(p, q)$, the latter problem is solved using an equivalence of categories due to Enright-Shelton. We also provide an explicit description of the non-zero spaces of intertwining operators in the exceptional cases, $E_{6}$ and $E_{7}$ (in the regular integral setting). A similar result for the remaining hermitian symmetric case, $G=\operatorname{SO}(2, n)$, was obtained previously in [2].

Associated to the pair $(G, K)$ there is a maximal parabolic subalgebra $\mathfrak{p}$ of $\mathfrak{g}$ (unexplained notation follows [3]), $\mathfrak{p}=\mathfrak{f} \oplus \mathfrak{g}$, where $\mathfrak{g}$ is the sum 
of the positive noncompact root spaces of $\mathfrak{g}$. For $\lambda \in \mathfrak{h}^{*}$ regular integral on $\mathfrak{f}$, we can define the generalized Verma module $V(\lambda)$ of highest weight $\lambda-\rho$ induced from $\mathfrak{p}$, and the associated Verma module $M(\lambda)$ and irreducible quotient $L(\lambda)$. Then it is well known (cf. [10]) that the space of covariant differential operators intertwining a pair of continuations of holomorphic discrete series for $G$, is isomorphic to $\operatorname{Hom}_{\mathfrak{g}}\left(V(\lambda), V\left(\lambda^{\prime}\right)\right)$, for appropriate $\lambda, \lambda^{\prime}$. Our answer to the classification problem will be given in this generalized Verma module setting. More precisely, we work in the category $\mathcal{O}(\mathfrak{g}, \mathfrak{p}, \chi)$ of all finitely generated $U(\mathfrak{g})$-modules which are $\mathfrak{p}$-finite, $\mathfrak{f}$-semisimple, and have generalized infinitesimal character $\chi$.

The problem of classifying homomorphisms between generalized Verma modules in the hermitian symmetric setting was first studied systematically by the first author. In his $\mathrm{Ph}$. D. thesis [1], he obtained all maps between so-called "scalar" modules (those induced from one-dimensional p-modules). This result was extended by Jakobsen [10] to include all maps whose domain or range is a scalar module. The current result completes the program in the regular integral case.

Crucial to all that follows is the structure of the Hasse diagram [7] $W^{K}$ attached to $(G, K)$ : this subset of $W$ parametrizes the generalized Verma modules having a given regular integral infinitesimal character. Following [3], having fixed such an infinitesimal character, we associate to each $w \in W^{K}$ a generalized Verma module $V_{w}$. Recall the arrow relation on $W^{K}: y \rightarrow w$ means $l(w)=l(y)+1$ and $w=y s_{\alpha}$ for some positive root $\alpha$. It turns out that, in the hermitian symmetric case, we may in fact always choose $\alpha$ simple; moreover, concrete models for the posets $W^{K}$ are known [7].
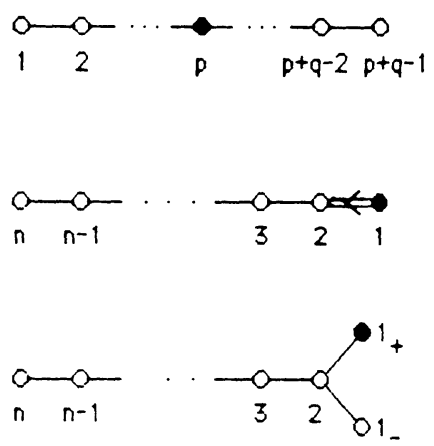

FIGURE 1 
We now describe these models in the cases of interest. Number the simple roots as indicated in Figure 1. For $\mathfrak{g}=\mathfrak{S} \mathfrak{u}(p, q)$,

$W^{K} \cong \mathscr{A}^{p, q}=\left\{\left(a_{1}, a_{2}, \ldots, a_{q}\right): p \geq a_{1} \geq a_{2} \geq \cdots \geq a_{q} \geq 0, a_{i} \in \mathbf{Z}\right\}$.

The arrow relations in $\mathscr{A}^{p, q}$ have the form $\left(a_{1}, \ldots, a_{i}-1, \ldots, a_{q}\right) \rightarrow$ $\left(a_{1}, \ldots, a_{i}, \ldots, a_{q}\right)$; the indicated arrow corresponds to reflection in the simple root $\alpha_{a_{t}+q-\imath}$.

For $\mathfrak{g}=\mathfrak{s} \mathfrak{p}(n, \mathbf{R})$ or $\mathfrak{s}^{*}(2(n+1))$,

$$
\begin{aligned}
W^{K} \cong \mathscr{C}^{n}=\left\{\left(a_{1}, a_{2}, \ldots, a_{n}\right): n \geq a_{1}\right. & \geq a_{2} \geq \cdots \geq a_{n} \geq 0, \\
a_{l}>a_{\imath+1} & \text { if } \left.a_{i}>0, a_{\imath} \in \mathbf{Z}\right\} .
\end{aligned}
$$

The arrow relations in $\mathscr{C}^{n}$ also have the form $\left(a_{1}, \ldots, a_{i}-1, \ldots, a_{n}\right) \rightarrow$ $\left(a_{1}, \ldots, a_{i}, \ldots, a_{n}\right)$. If $a_{i}>1$ or $\mathfrak{g}=\mathfrak{s p}(n, \mathbf{R})$, the indicated arrow corresponds to reflection in the simple root $\alpha_{a_{i}}$. If $a_{i}=1$ and $\mathfrak{g}=$ $\mathfrak{S O}^{*}(2(n+1))$, the reflection is in $\alpha_{1_{+}}$if $n-i$ is even, and in $\alpha_{1_{-}}$if $n-i$ is odd.

(1.1) Definition. For $W^{K} \cong \mathscr{A}^{p, q}$ or $\mathscr{C}^{n}$, whenever $w^{\prime} \equiv\left(\cdots, a_{\imath}-\right.$ $1, \cdots) \rightarrow\left(\cdots, a_{i}, \cdots\right) \equiv w$ is effected by the simple root $\alpha$ (with $w, w^{\prime} \in W^{K}$ ), we shall say that $\alpha$ acts on $w^{\prime}$ and $w$ at coordinate $i$.

For $g$ of type $E_{6}$ or $E_{7}$, the partially ordered sets $W^{K}$ are given in [4], and may also be distilled from Figures 2 and 3.

2. The classical cases. We first treat the "standard maps" [2]. Given $w \in W^{K}, w=\left(a_{1}, \ldots, a_{n}\right)$, set $\mathscr{I}=\left\{1 \leq i \leq n:\left(a_{1}, \ldots, a_{i}-\right.\right.$ $\left.\left.1, \ldots, a_{n}\right) \in W^{K}\right)$. Now if $x \in W^{K}, x=\left(b_{1}, \ldots, b_{n}\right)$, we say $x$ is standard with respect to $w$ if, for $1 \leq i \leq n$, either $b_{i}=a_{i}$ or $b_{i}=a_{i}-1$, with $b_{\imath}=a_{i}-1$ only if $i \in \mathscr{I}$. By [8, Lemma 4.2] we have:

(2.1) LEMMA. If $x$ is standard with respect to $w$, then there is a non-zero standard homomorphism $V_{x} \rightarrow V_{w}$, which is unique up to scalar multiple.

We now state some necessary conditions for the existence of non-zero maps in general.

(2.2) Lemma [6]. Suppose $w>w s_{\alpha} \equiv w^{\prime}$ with $w, w^{\prime} \in W^{K}, \alpha$ simple. Then there is a short exact sequence

$$
0 \rightarrow V_{w} \rightarrow \varphi_{\alpha} \psi_{\alpha} V_{w} \rightarrow V_{w^{\prime}} \rightarrow 0
$$

(where $\varphi_{\alpha}, \psi_{\alpha}$ are translation functors). 
(2.3) Corollary (of (2.2), [3, (2.11)]. For $x, w \in W^{K}, \operatorname{Hom}_{\mathfrak{g}}\left(V_{x}, V_{w}\right)$ $=0$ if there is a simple root $\alpha$ such that either

(a) $w>w s_{\alpha} \in W^{K}$ but $\alpha$ does not act on $x$,

or $\quad$ (b) $x<x s_{\alpha} \in W^{K}$ but $\alpha$ does not act on $w$.

We can now state our main result.

(2.4) Theorem. Fix $G$ to be one of $\mathrm{SU}(p, q), \mathrm{Sp}(n, \mathbf{R})$, or $\mathrm{SO}^{*}(2 n)$, and fix a regular integral infinitesimal character. Suppose that $x, w \in W^{K}$, $x \leq w$, and assume that there is no simple root $\alpha$ satisfying condition (a) or (b) of Corollary 2.3. Then either $(x, w)$ is a standard pair (so $\operatorname{Hom}_{\mathfrak{g}}\left(V_{x}, V_{w}\right) \neq 0$ by Lemma 2.1), or there is a simple root $\alpha$ (which can be determined explicitly) such that $\operatorname{Hom}_{\mathfrak{g}}\left(V_{x}, V_{w}\right)=\operatorname{Hom}_{\mathfrak{g}}\left(\psi_{\alpha} V_{x}, \psi_{\alpha} V_{w}\right)$. In either case, non-zero homomorphisms are unique up to scalar multiple.

We first prove some technical lemmas concerning the combinatorial aspects of $W^{K}$. While the statements of the lemmas are true for all $G$, we shall prove them and the theorem only for $G=\mathrm{SU}(p, q)$. The arguments for the other cases are similar, requiring minor modifications only as dictated by the different structure of $W^{K}$. For the remainder of this section, we shall assume that $\mathfrak{g}=\mathfrak{g} \mathfrak{u}(p, q)$.

(2.5) Lemma. Fix $w \in W^{K}$, and suppose that $\alpha(1), \alpha(2)$ are simple roots acting on coordinates $i(1), i(2)$ in $w$. Assume that $i(1) \leq i(2)$, and if $i(1)=i(2)$, then $w s_{\alpha(2)}<w<w s_{\alpha(1)}$. Then $\alpha(1)=\alpha_{j_{1}}, \alpha(2)=\alpha_{j_{2}}$, with $j_{1}>j_{2}$ (in the ordering of Fig. 1).

Proof. Let $w=\left(a_{1}, \ldots, a_{q}\right)$. Then $j_{1}=a_{i(1)}+q-i(1)+\delta_{1}, j_{2}=$ $a_{i(2)}+q-i(2)+\delta_{2}, \delta_{1}, \delta_{2} \in\{0,1\}$. Hence

$$
j_{1}-j_{2}=\left(a_{i(1)}-a_{l(2)}\right)+(i(2)-i(1))+\left(\delta_{1}-\delta_{2}\right) .
$$

Now $i(1) \leq i(2)$ implies $a_{i(1)} \geq \mathrm{a}_{i(2)}$. Moreover, if $a_{i(1)}=a_{i(2)}$ then $\delta_{1}=1$ and $\delta_{2}=0$. Thus $j_{1}-j_{2}>0$.

(2.6) Lemma. Let $x, w \in W^{K}, x \leq w$. Let $\alpha$ be a simple root such that $x s_{\alpha}, w s_{\alpha} \in W^{K}$. Suppose $\alpha$ acts on coordinate $i$ in $x$ and coordinate $j$ in $w$. Then $i \leq j$.

Proof. Set $x=\left(b_{1}, \ldots, b_{q}\right), w=\left(a_{1}, \ldots, a_{q}\right)$, with $b_{k} \leq a_{k}, 1 \leq k \leq$ $q$. We have

$$
b_{i}+q-i+\delta_{i}=a_{j}+q-j+\delta_{j} \quad\left(\delta_{i}, \delta_{j} \in\{0,1\}\right) .
$$


Suppose $i>j$. Then $b_{i} \leq b_{j} \leq a_{j}$. On the other hand, $b_{i}=a_{j}+(i-j)+$ $\left(\delta_{j}-\delta_{i}\right)$, forcing $i=j+1, \delta_{j}=0, \delta_{i}=1, b_{j+1}=a_{j}$. But $\delta_{j}=0$ implies $a_{j+1}<a_{j}=b_{j+1}$, a contradiction.

(2.7) Lemma. Let $x, w \in W^{K}, x \leq w$. Assume that, for each simple root $\alpha$ such that $x<x s_{\alpha} \in W^{K}, \alpha$ acts on the same coordinate in $w$ as it does in $x$. Then $x$ is standard with respect to $w$.

Proof. Let

$$
x=\left(b_{i(1)}, \ldots, b_{i(1)}, b_{i(2)}, \ldots, b_{i(2)}, \ldots, b_{i(m)}, \ldots, b_{i(m)}\right)
$$

where $b_{i(1)}>b_{i(2)}>\cdots>b_{i(m)}$, and $i(k)$ is the index of the first occurrence of $b_{i(k)}$. If $w=\left(a_{1}, \ldots, a_{q}\right)$, then our hypothesis implies that $a_{i(k)}=b_{i(k)}+\delta_{k}, \delta_{k} \in\{0,1\}, 1 \leq k \leq m$; moreover, if $\delta_{k}=1$ then $a_{i(k)+1}<a_{i(k)}$. But this, together with the assumption $x \leq w$, implies that $w=\left(b_{i(1)}+\delta_{1}, b_{i(1)}, \ldots, b_{i(1)}, b_{i(2)}+\delta_{2}, b_{i(2)}, \ldots, b_{i(2)}, \ldots, b_{i(m)}+\delta_{m}\right.$,

$$
\left.b_{i(m)}, \ldots, b_{i(m)}\right) \text {. }
$$

Hence $x$ is standard with respect to $w$.

Proof of Theorem 2.4. We continue to assume $\mathfrak{g}=\mathfrak{s} \mathfrak{u}(p, q)$. Let $x$ have the form (2.8). Denote by $\alpha(k)$ the simple root which acts on $x$ by sending $b_{i(k)}$ to $b_{i(k)}+1$. By hypothesis, each $\alpha(k)$ acts on $w$. By Lemma 2.7, we may assume there is some $k$ for which $\alpha(k)$ does not act on coordinate $i(k)$ in $w$; choose $k$ minimal with this property. Set $\alpha=\alpha(k)$. By Lemma 2.6, $\alpha$ must act on a coordinate $a_{s}$ in $w$ with $s>i(k)$. Thus

$$
\begin{aligned}
w=\left(b_{i(1)}+\delta_{1}, b_{i(1)}, \ldots, b_{i(1)}, \ldots, b_{i(k-1)}+\delta_{k-1},\right. & \\
& \left.b_{i(k-1)}, \ldots, b_{i(k-1)}, a_{i(k)}, a_{i(k)+1}, \ldots, a_{s-1}, a_{s}, \cdots\right)
\end{aligned}
$$

where $a_{s}=b_{i(k)}+s-i(k)+\delta_{k}, \delta_{j} \in\{0,1\}, 1 \leq j \leq k$.

We claim that $a_{s-1}=a_{s}$. To see this, denote by $\beta(j)$ the simple root which acts on $x$ by sending $b_{i(j)}$ to $b_{i(j)}-1$ in coordinate $i(j+1)-1$. For $1 \leq j<k$, it is clear from Lemma 2.6 and (2.9) that if $W^{K} \ni w s_{\beta(j)}$ $\rightarrow w, \beta(j)$ must send $b_{i(j)}$ to $b_{i(j)}-1$ in coordinate $i(j+1)-1$. Thus if $a_{s-1}>a_{s}$, then the simple reflection $\beta$ which sends $a_{s-1}$ to $a_{s-1}-1$ in $w$ cannot be $\beta(j)$ for $1 \leq j<k$; it also is not $\alpha(j)$ for $1 \leq j<k$. But since $\beta$ acts to the left of $\alpha(k)$ in $w$, Lemma 2.5 implies that $\beta$ cannot act on $x$. This contradicts our hypothesis concerning Corollary 2.3(a).

Thus $a_{s-1}=a_{s}$, implying that $\delta_{k}=1$. Hence $w^{\prime} \equiv w s_{\alpha(k)} \rightarrow w$. But now $w^{\prime} s_{\beta} \rightarrow w^{\prime}$ (where $\beta$ is defined in the preceding paragraph), so again using Corollary 2.3(a), we have $\operatorname{Hom}_{\mathfrak{g}}\left(V_{x}, V_{w^{\prime}}\right)=0$. Applying $\operatorname{Hom}_{\mathfrak{g}}\left(V_{x},-\right)$ 
to the exact sequence in Lemma 2.2 gives

$$
\operatorname{Hom}_{\mathfrak{g}}\left(V_{x}, V_{w}\right) \cong \operatorname{Hom}_{\mathfrak{g}}\left(V_{x}, \varphi_{\alpha} \psi_{\alpha} V_{w}\right) \cong \operatorname{Hom}_{\mathfrak{g}}\left(\psi_{\alpha} V_{x}, \psi_{\alpha} V_{w}\right) \text {. }
$$

(2.10) Corollary. In case $G=\mathrm{SU}(p, q)$, the spaces of homomorphisms between generalized Verma modules having regular integral infinitesimal character are at most one-dimensional, and can be computed inductively. That is, if the result is known for all $\mathrm{SU}\left(p^{\prime}, q^{\prime}\right), p^{\prime} \leq p, q^{\prime} \leq q$, with at least one inequality strict, then the result for $\mathrm{SU}(p, q)$ follows. ${ }^{1}$

Proof. This is a direct consequence of the theorem, and the following result.

(2.11) THEOREM (Enright-Shelton [5]). There is a natural equivalence of categories between $\mathcal{O}\left(\mathfrak{g} \mathfrak{H}(p-1, q-1), \mathfrak{p}^{\prime}\right.$, triv) and $\mathcal{O}(\mathfrak{B} \mathfrak{H}(p, q), \mathfrak{p}, \lambda)$, where $\lambda$ is the semi-regular weight obtained by pushing $\rho$ to any one simple root wall.

3. The exceptional cases. Let $(G, K)$ correspond to an exceptional $E_{6}$ or $E_{7}$ case. We sketch the argument for computing all homomorphisms between the generalized Verma modules in this case.

Step 1. Given $w \in W^{K}$, compute the composition factors of $V_{w}$. In fact, we can compute a $\mathrm{g}$-filtration of $V_{w}$ with semisimple subquotients; for details, see [4] or [9]. In particular, as noted by Irving [9], $V_{w}$ has an irreducible socle $L_{w^{*}}$; recall, socle $V_{w}=$ largest semisimple submodule of $V_{w}$.

Step 2. Fix $V_{w}$ and assume we understand $H^{0}\left(\xi, V_{w^{\prime}}\right)$ for all $w^{\prime}<w$; i.e., we start from the irreducible $V_{e}$ and "work up" the diagram. From step 1, we have

$$
V_{w}=L_{y_{1}}+\cdots+L_{y_{n}}+L_{z_{1}}+\cdots+L_{z_{m}}
$$

in the Grothendieck group. Furthermore, this indexing is set up so that $L_{y_{1}}, \ldots, L_{y_{n}}$ correspond to non-zero standard maps $V_{y_{t}} \rightarrow V_{w}$; these are settled as in Lemma 2.1. It remains to determine if $L_{z_{l}}$ corresponds to a non-zero map $V_{z_{t}} \rightarrow V_{w}$. To do this we proceed case-by-case, in the $z_{i}$ 's. First of all (as noted in step 1), socle $V_{w}=L_{w^{\#}}$. Obviously there is a non-zero map $V_{w^{\#}} \rightarrow V_{w}$. A key observation is:

(3.1) If $z_{i} \ngtr w^{\#}$, then there does not exist a non-zero map $V_{z_{i}} \rightarrow V_{w}$.

Using (3.1), we can typically resolve that several of the factors $L_{z_{l}}$ do not

\footnotetext{
${ }^{1}$ Added in proof: The first author and T. Enright have recently obtained a closed form description of the homomorphisms for $\mathrm{SU}(p, q)$.
} 
correspond to maps. This leaves a few $L_{z_{t}}$ 's to work with. These are handled using $\operatorname{Hom}\left(V_{z_{i}},-\right)$ applied to appropriate sequences, as in Lemma 2.2. Although we do not have results as in [5] at our disposal, the singular modules $\psi_{\alpha} V_{z_{2}}$ and $\psi_{\alpha} V_{w}$ are sufficiently simple to detect their $\mathfrak{g}$-invariants; typically, they have just two composition factors. We leave it to the (ambitious) reader to fill in the details from here.

We have now proved

(3.2) THEOREM. The homomorphisms between generalized Verma modules for $E_{6}$ and $E_{7}$ are known and are tabulated in Figures 2 and 3, and Table 1 .

(3.3) Explanation of figures and table. In Figures 2 and 3, we have reproduced $W^{K}$ for $E_{6}$ and $E_{7}$. The elements of $W^{K}$ are numbered sequentially. In Figure 2, all non-zero homomorphisms $V_{x} \rightarrow V_{w}$ are indicated. For $E_{7}$, only the non-zero standard maps are indicated in Figure 3; the non-standard homomorphisms are listed in Table 1. The

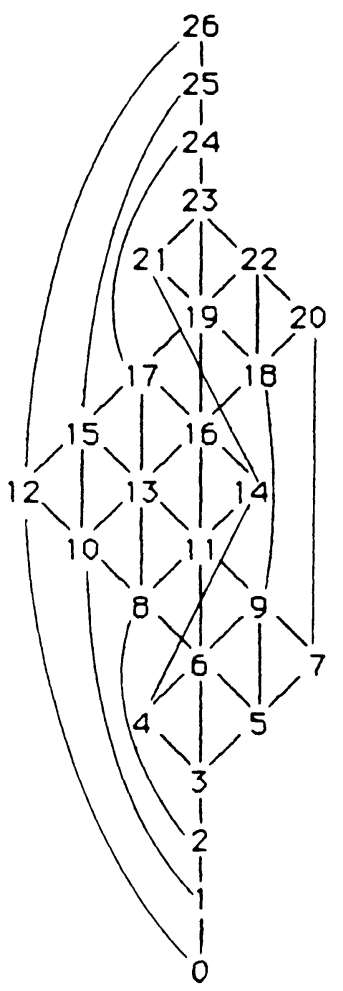

FIGURE 2

Maps between generalized Verma modules for $E_{6}$ 


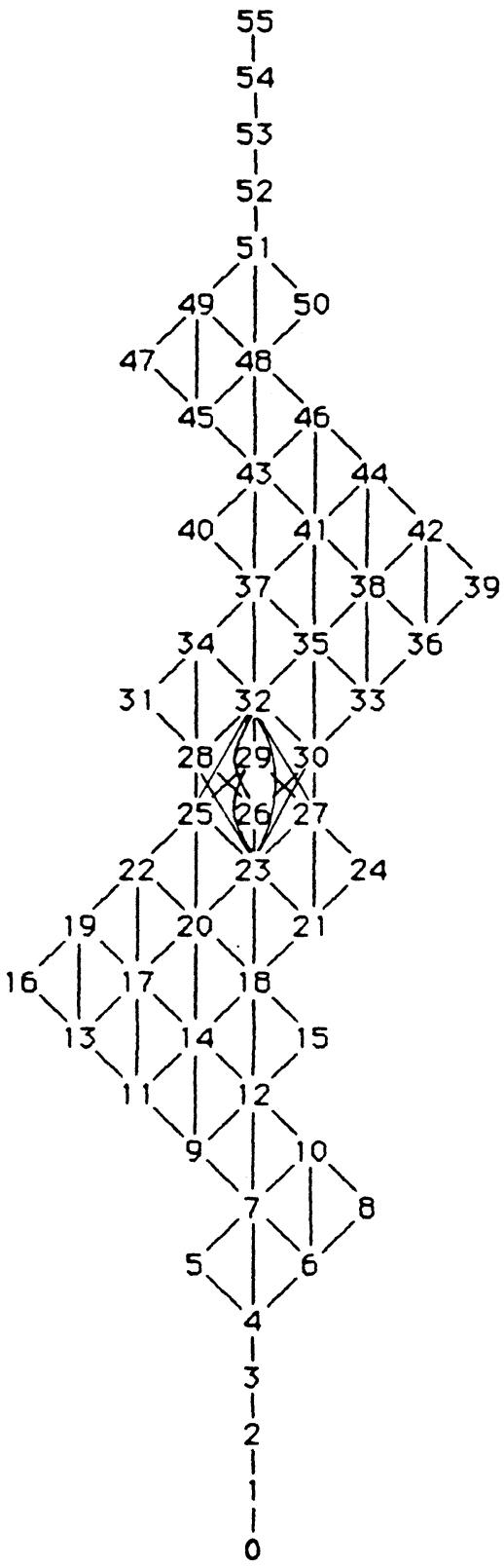

Figure 3

Standard maps between generalized Verma modules for $E_{7}$ 


\begin{tabular}{|c|c|c|c|}
\hline$x$ & $w$ & $x$ & $w$ \\
\hline 0 & 16,55 & 16 & 42 \\
\hline 1 & 13,54 & 17 & 36,38 \\
\hline 2 & 11,53 & 18 & 37 \\
\hline 3 & 9,52 & 19 & 38 \\
\hline 4 & 51 & 20 & 33,35 \\
\hline 5 & 15,50 & 21 & 31,34 \\
\hline 6 & 49 & 22 & 35 \\
\hline 7 & 48 & 24 & 34 \\
\hline 8 & 24,47 & 29 & 40 \\
\hline 9 & 46 & 31 & 47 \\
\hline 10 & 21,45 & 34 & 45 \\
\hline 11 & 44 & 39 & 55 \\
\hline 12 & 43 & 40 & 50 \\
\hline 13 & 39,42 & 42 & 54 \\
\hline 14 & 41 & 44 & 53 \\
\hline 15 & 26,40 & 46 & 52 \\
\hline
\end{tabular}

TABLE 1

Non-standard maps for $E_{7}$.

parametrization of Figure 3 is used. The first column gives $x$, and the second column all $w$, such that $\operatorname{Hom}_{\mathfrak{g}}\left(V_{x}, V_{w}\right) \neq 0$.

4. Two classical examples. In this section, we present the results of applying the algorithm $(2.4)$, in case $G=\mathrm{SU}(3,3)$ or $\mathrm{SO}^{*}(10)$. The partially ordered sets $W^{K}$, together with all maps, are given by Figures 4 and 5. Note that $W^{K}$ contains subdiagrams "at the bottom" isomorphic in an obvious fashion to $W^{K}$ for lower rank groups: either $\operatorname{SU}(p, q)$ with $p$, $q \leq 3$, or $\mathrm{SO}^{*}(2 n)$ with $n<5$. Moreover, there are equivalences analogous to [3, (2.6)], which means that the maps for these lower rank groups can be read off from the corresponding subdiagrams. The same ideas apply to $\mathrm{Sp}(4)$, leading to a diagram isomorphic to Figure 5. 


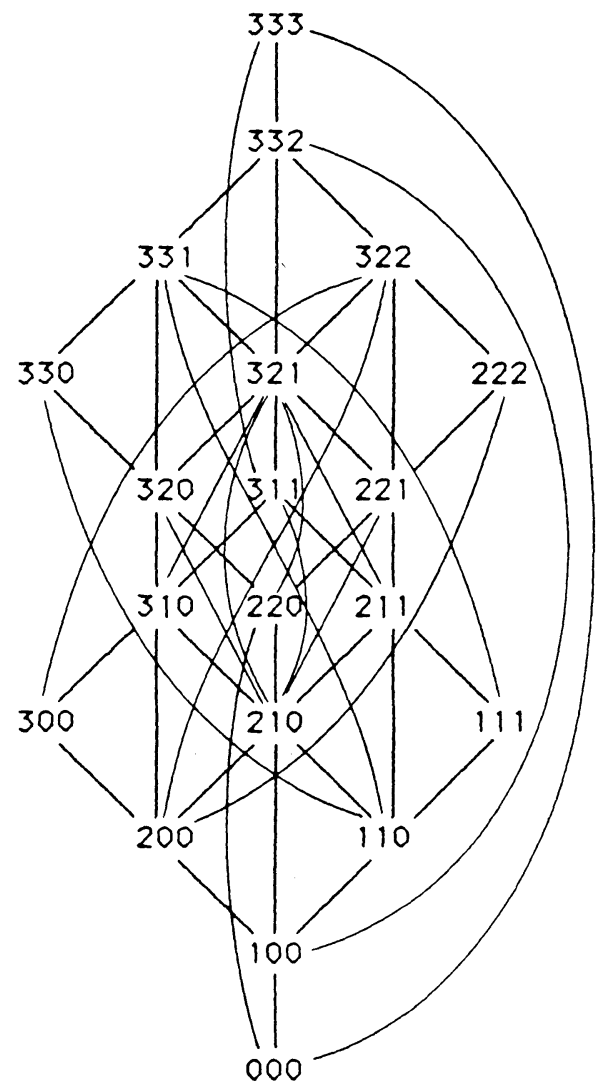

FIGURE 4

Maps between generalized Verma modules for $\operatorname{SU}(3,3)$ 


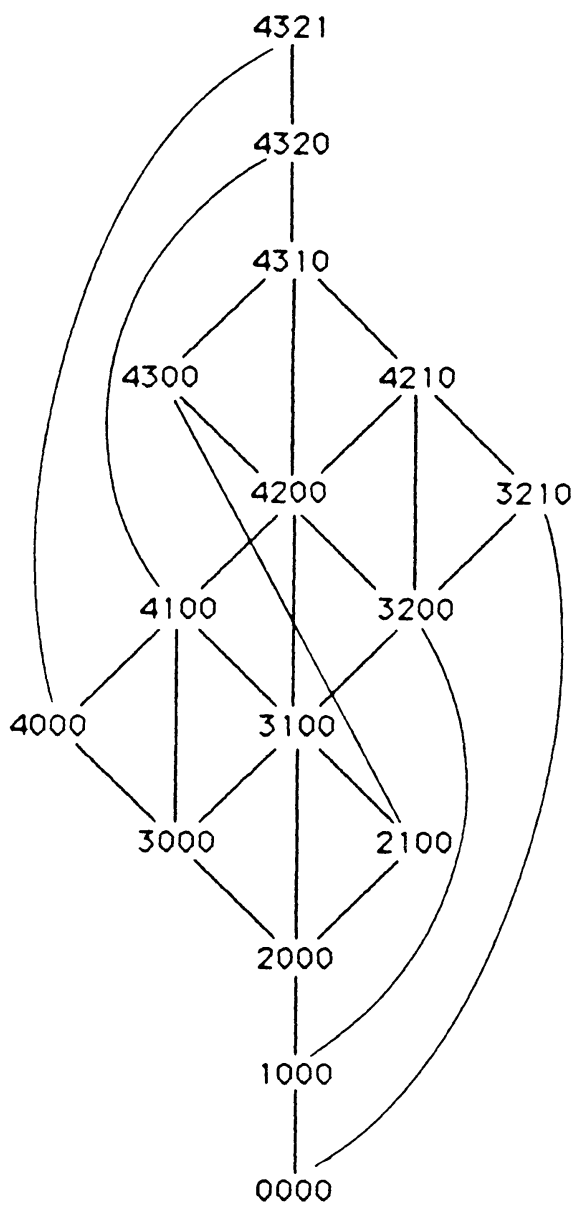

Figure 5

Maps between generalized Verma modules for $\mathrm{SO}^{*}(10)$ 


\section{REFERENCES}

[1] B. D. Boe, Homomorphisms between generalized Verma modules, Trans. Amer. Math. Soc., 208 (1985), 791-799.

[2] B. D. Boe and D. H. Collingwood, A comparison theory for the structure of induced representations, J. Algebra, 94 (1985), 511-545.

[3] _ A multiplicity one theorem for holomorphically induced representations, Math. Zeit., (to appear).

[4] D. H. Collingwood, The n-homology of Harish-Chandra modules I: Generalizing a theorem of Kostant, Math. Ann., 272 (1985), 161-187.

[5] T. Enright and B. Shelton, Categories of highest weight modules: applications to $\mathrm{SU}(p, q)$, preprint, 1985 .

[6] O. Gabber and A. Joseph, Towards the Kazhdan-Lusztig conjectures, Ann. Scient. Ec. Norm. Sup., 14 (1981), 261-302.

[7] H. Hiller, Geometry of Coxeter Groups, Research notes in mathematics, Pitman, Boston, 1982.

[8] R. Irving, Projective modules in the category $\mathcal{O}_{s}$ : Loewy series, Trans. Amer. Math. Soc., 291 (1985), 733-754.

[9] , Projective modules in the category $\mathcal{O}_{s}$ : Self-duality, Trans. Amer. Math. Soc., 291 (1985), 701-732.

[10] H. P. Jakobsen, Basic covariant differential operators on hermitian symmetric spaces, Ann. Scient. Ec. Norm. Sup., (to appear).

Received April 2, 1985. The first author was supported in part by NSF Grant No. DMS 85-03781. The second author was supported by NSF Postdoctoral Fellowship No. MCS 85-11467.

The UNIVERSITY OF CALIFORNIA, BERKeley

BERKELEY, CA 94720

AND

UNIVERSITY OF OREGON

EUGENE, OR 97403 


\section{PACIFIC JOURNAL OF MATHEMATICS EDITORS}

\author{
V. S. VARADARAJAN \\ (Managing Editor) \\ University of California \\ Los Angeles, CA 90024 \\ HeRbert Clemens \\ University of Utah \\ Salt Lake City, UT 84112 \\ R. FINN \\ Stanford University \\ Stanford, CA 94305
}

HERMANN FLASCHKA

University of Arizona

Tucson, AZ 85721

RAMESH A. GANGOLLI

University of Washington

Seattle, WA 98195

VAUGHAN F. R. JONES

University of California

Berkeley, CA 94720

ROBION KIRBY

University of California

Berkeley, CA 94720
C. C. MOORE

University of California

Berkeley, CA 94720

H. SAMELSON

Stanford University

Stanford, CA 94305

HAROLD STARK

University of California, San Diego

La Jolla, CA 92093

\section{ASSOCIATE EDITORS}
R. Arens
E. F. BECKENBACH
B. H. NEUMANN
F. WOLF
K. YoshIDA (1906-1982)

\section{SUPPORTING INSTITUTIONS}

UNIVERSITY OF ARIZONA

UNIVERSITY OF BRITISH COLUMBIA

CALIFORNIA INSTITUTE OF TECHNOLOGY

UNIVERSITY OF CALIFORNIA

MONTANA STATE UNIVERSITY

UNIVERSITY OF NEVADA, RENO

NEW MEXICO STATE UNIVERSITY

OREGON STATE UNIVERSITY
UNIVERSITY OF OREGON

UNIVERSITY OF SOUTHERN CALIFORNIA

TANFORD UNIVERSITY

UNIVERSITY OF HAWAII

UNIVERSITY OF TOKYO

UNIVERSITY OF UTAH

WASHINGTON STATE UNIVERSITY

UNIVERSITY OF WASHINGTON 


\section{Pacific Journal of Mathematics}

Vol. 124, No. $1 \quad$ May, 1986

Kinetsu Abe and Martin Andrew Magid, Relative nullity foliations and indefinite isometric immersions

Erik P. van den Ban, A convexity theorem for semisimple symmetric

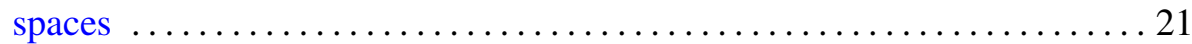

Bo Berndtsson and Thomas Joseph Ransford, Analytic multifunctions, the $\bar{\partial}$-equation, and a proof of the corona theorem .................. 57

Brian Boe and David H. Collingwood, Intertwining operators between

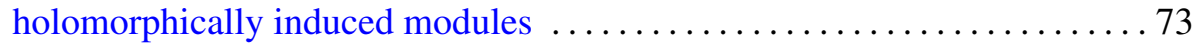

Giuseppe Ceresa and Alessandro Verra, The Abel-Jacobi isomorphism for

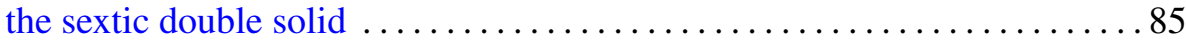

Kun Soo Chang, Jae Moon Ahn and Joo Sup Chang, An evaluation of the conditional Yeh-Wiener integral ........................... 107

Charles Dale Frohman, Minimal surfaces and Heegaard splittings of the

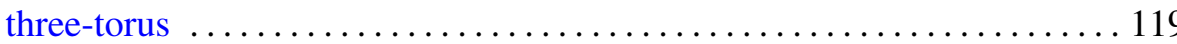

Robert M. Guralnick, Power cancellation of modules $\ldots \ldots \ldots \ldots \ldots \ldots 131$

Kenneth Hardy and Kenneth S. Williams, On the solvability of the Diophantine equation $d V^{2}-2 e V W-d W^{2}=1 \ldots \ldots \ldots \ldots \ldots \ldots \ldots \ldots \ldots$

Ray Alden Kunze and Stephen Scheinberg, Alternative algebras having

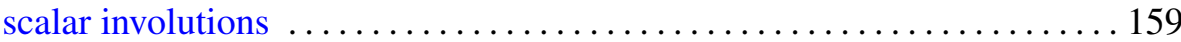

W. B. Raymond Lickorish and Kenneth Millett, The reversing result for the Jones polynomial $\ldots \ldots \ldots \ldots \ldots \ldots \ldots \ldots \ldots$

Guido Lupacciolu, A theorem on holomorphic extension of CR-functions

William Schumacher Massey and Lorenzo Traldi, On a conjecture of K. Murasugi

Dinakar Ramakrishnan, Spectral decomposition of $L^{2}(N \backslash \mathrm{GL}(2), \eta)$

Steven L. Sperber, On solutions of differential equations which satisfy certain algebraic relations 